\section{Routine primary immunisation using a longer needle resulted in fewer local reactions in infants}

Diggle L, Deeks J. Effect of needle length on incidence of local reactions to routine immunisation in infants aged 4 months: randomised controlled trial. BMJ 2000 Oct 14;321:931-3.

\section{QUESTION: When giving routine immunisations to infants, does needle length affect the incidence of local reactions?}

\section{Design}

Randomised (allocation concealed), blinded (outcome assessors), controlled trial with follow up to 3 days.

\section{Setting}

8 general practices in Buckinghamshire, UK.

\section{Participants}

119 healthy infants attending routine immunisation clinics. Exclusion criteria were those applicable to children receiving primary immunisations. 92\% of infants ( $58 \%$ boys) completed follow up.

\section{Intervention}

58 infants were allocated to receive their third dose of diphtheria, pertussis, and tetanus and Haemophilus influenzae type b vaccines (due at 16 weeks) given with a 23 gauge, $25 \mathrm{~mm}$ (longer) blue hub needle and 61 were allocated to vaccine administration with a 25 gauge, 16 $\mathrm{mm}$ (shorter) orange hub needle. Practice nurses were instructed to inject into the anterolateral thigh, stretching the skin taut and inserting the needle at a $90^{\circ}$ angle to the skin up to the hub.

\section{Main outcome measures}

Parent recording of redness, swelling, and tenderness at 6 hours, and at 1, 2, and 3 days after immunisation.

\section{Main results}

Infants who were vaccinated with the longer needle had lower rates of any local reaction compared with infants vaccinated with the shorter needle; specifically, infants vaccinated with the longer needle had lower rates of redness and swelling at 6 hours, and at 1, 2, and 3 days (table). The groups did not differ significantly for tenderness at any time point.

\section{Conclusion}

Infants who had their 16 week primary immunisation given with a longer (23 gauge, $25 \mathrm{~mm}$ ) needle had lower rates of local redness and swelling for up to 3 days compared with those who had immunisations given with a shorter (25 gauge, $16 \mathrm{~mm}$ ) needle.
Longer $v$ shorter needle for giving routine immunisations to infants*

\begin{tabular}{|c|c|c|c|c|}
\hline Outcomes & Longer needle & Shorter needle & $\operatorname{RRR}(95 \% \mathrm{Cl})$ & NNT (Cl) \\
\hline $\begin{array}{l}\text { Any local reaction up } \\
\text { to } 3 \text { days }\end{array}$ & $62 \%$ & $84 \%$ & $26 \%$ (7 to 43$)$ & 5 (3 to 19$)$ \\
\hline Redness at 6 hours & $40 \%$ & $60 \%$ & $34 \%$ (3 to 56$)$ & 5 (3 to 81$)$ \\
\hline At 1 day & $28 \%$ & $63 \%$ & $55 \%$ (30 to 73$)$ & $3(2$ to 7$)$ \\
\hline At 2 days & $9 \%$ & $39 \%$ & $76 \%$ (43 to 90$)$ & 4 (3 to 8$)$ \\
\hline At 3 days & $4 \%$ & $28 \%$ & $87 \%$ (51 to 96$)$ & 5 (3 to 9$)$ \\
\hline Swelling at 6 hours & $23 \%$ & $58 \%$ & $61 \%(35$ to 78$)$ & $3(2$ to 6$)$ \\
\hline At 1 day & $28 \%$ & $63 \%$ & $55 \%$ (30 to 73$)$ & $3(2$ to 7$)$ \\
\hline At 2 days & $19 \%$ & $51 \%$ & $63 \%(34$ to 80$)$ & 4 (3 to 7$)$ \\
\hline At 3 days & $13 \%$ & $40 \%$ & $67 \%$ (33 to 85$)$ & 4 (3 to 10$)$ \\
\hline
\end{tabular}

*Abbreviations defined in glossary; RRR, NNT, and $\mathrm{Cl}$ calculated from data in article.

\section{COMMENTARY}

What a wonderful example of evidence-based nursing in action! This study by Diggle and Deeks was done in response to requests from nurses in the UK for evidence on which to base immunisation practice. It is the first randomised controlled trial to evaluate the relation between needle length and local reactions when immunising infants. Ipp et al assessed the effects of both injection site and needle length on adverse reactions to diphtheria, pertussis, tetanus, and polio vaccination in 18 month old infants. ${ }^{1}$ Although this was not a randomised trial, their finding that injection into the thigh with a longer needle was associated with less redness and swelling supports the findings of Diggle and Deeks.

This trial was done in several general practices in the UK, which increases the generalisability of the findings. Potential biases were avoided by concealing patient allocation to treatment groups in sequentially numbered, opaque envelopes and by keeping parents, who recorded the outcomes, blind to which needle length was used to vaccinate their child. The study could have been strengthened by standardised assessment of outcome by the same individual for all infants and by consideration in the analyses of other factors that might have influenced local reactions such as percentage of body fat.

This study has clinical relevance for practitioners responsible for vaccinating infants. Both redness and swelling were significantly reduced when using the longer needle. The differences suggest that, for every 3-5 infants vaccinated with the longer rather than the shorter needle, 1 case of redness and 1 case of swelling would be prevented. In a time when the public has limited knowledge of the risks associated with contracting an infectious disease, minimising the adverse events accompanying infant vaccination could enhance public acceptance of immunisation.

Colleen Van Berkel, RN, MHSc Program Manager, Communicable Disease Control City of Hamilton Hamilton, Ontario, Canada

1 Ipp MM, Gold R, Goldbach M, et al. Adverse reactions to diphtheria, tetanus, pertussis-polio vaccination at 18 months of age: effect of injection site and needle length. Pediatrics 1989;83:679-82. 